\title{
The Inheritance and Development of Contemporary Mogu Flower-bird Painting
}

\author{
Hao Xiaofei \\ Department of Chinese Painting, Hubei Institute of Fine Arts Wuhan, Hubei 430070, China
}

745282008@qq.com

Keywords: Mogu flower-bird painting; tradition; innovation

\begin{abstract}
Mogu is a special expression technique between freehand brushwork and fine brushwork with a long history line. The contemporary mogu flower-bird painting has shown a variety of developmental status. Artists are exploring the painting language, techniques, and media on the basis of taking advantage of traditional mogu flower-bird painting, making this ancient painting species a new glow.
\end{abstract}

\section{Introduction}

"Mogu method" is a landmark innovation in the history of Chinese traditional flower and bird painting. The so-called mogu is "do not use pen and ink, just use color to paint flowers." The outline of objects is not drawn and the objects are depicted directly in color, whose essence lies in the organic combination of wielding the pen and coloring, and the requirement of the painter to have a well-thought-out plan to strike while the iron is hot. The word "mogu painting" was first seen in "Painting knowledge records·mogu painting" of Guo Ruoxu from the Northern Song Dynasty: "When Xu Chongsi paints the mogu painting, he is known for no pen and ink, but his painting is definitely colorful." This is the first time in the history of painting to define the "mogu painting" and it is also the first visible record of "mogu".

This painting method is to remove the "line" based on the painting technique, which further tests the ability of form and structure cognition and perception control during painting. Its effect is more abundant than the traditional "drawing the outline of flower and adding details to the leaf in Chinese ink", and it also tests the painter's understanding of the color perception and texture of the objects he depicts. The expression is more abundant and the dynamics and appearance of the object can be more highlighted.

After the mogu flower-bird painting appeared in the Five Dynasties and the Northern Song Dynasty, it has developed in different degrees in Yuan and Ming Dynasty. As is recorded in "The famous paintings of one's own royal court" that "Xu Xi is good at painting flower, bamboo, forests, cicada, frog, grass and worm and the like. His intention is beyond the ancients, and he had creative method, and was especially good at coloring, which could bring absolute vitality." "His intention is beyond the ancients" means that he didn't paint in the ancients' method. This book has another record for Xu Xi's paintings: "Xu Xi's method is very unique, which uses the Chinese ink to paint the branches and leaves, bud and sepal first and then color them. Therefore, its artistic conception and style was set previously, which was not very far from the nature. So it is appropriate to regard it as the crown of the world, superb work." So the mogu painting of Xu Chongsi should be from his grandfather $\mathrm{Xu} \mathrm{Xi}$. During this period, the painter who enjoyed equal popularity with him is Zhao Chang. Zhao Chang's first teacher was Teng Changyou, and later on he followed the mogu painting of Xu Chongsi. Oftentimes, in the early morning when the dew way still not dry, he observed the demeanor of the grass and trees in the flower garden and named himself "Zhao Chang paint from life”.

In the Yuan Dynasty, social politics was ruled by people not of the same clan. The neat and magnificent imperial court technique formed by the court painting institute since the Song Dynasty declined. Most of the literati lived in seclusion. The imperial court technique was replaced by the freehand literati painting, and the representatives are Qian Xuan,Wang Yuan and other painters. The 
painting of Qian Xuan has a prominent style of wildness and naturalness, and it uses more techniques of rinsing ink with water, which is more expressive in form.

On the basis of inheriting the fine brushwork and mogu painting of people in the Song Dynasty, Sun Long, a court painter of the Ming Dynasty absorbed the essence of painting techniques from various schools and formed a new technique of mogu flower-bird painting. With the liberation of imprisonment for the use of color by the painting circle, the color of the mogu flower-bird painting gradually became more abundant. At the same time, there were also painters such as Shen Zhou, Chen Chun, Xu Wei, etc, who served as a link between past and future in the history of Chinese traditional flower and bird painting.

In the early Qing Dynasty, Yun Shouping "studied the ancient and modern painting to follow Xu Chongsi in the Northern Song Dynasty and created his unique method to become a decent school." "Every time when he painted a flower, he must break it off to insert it in a bottle and depicted it utmostly to try his best to make it lively and colourful." His painting method is "painting with colors directly and then repeated it to make it complete." On the basis of the mogu painting of the Northern Song Dynasty, he created a new style of "Yun style" mogu flower painting, which has free technique of writing, bright and clean color and elegant style. The "Changzhou painting school" represented by Yun Shouping has vigorously promoted the mogu flower-bird painting. So far, the techniques and performance of the mogu flower-bird painting have become very mature and they have entered an unprecedented period of prosperity.

In the Qing Dynasty, after Yun Shouping, the most representative painters were Ren Bonian, Xu $\mathrm{Gu}$, Ju Lian, and Ju Chao and other painters. After influenced by the Western thought, the early modern Chinese flower and bird painting showed a variety of development directions. The painters are constantly absorbing, blending and refining the traditional techniques and the emerging western painting techniques.

\section{The Main Technique Types of Traditional "Mogu Method"}

"Mogu method" is a way of painting between the fine brushwork and freehand brushwork. The delicacy school is close to the fine brushwork, and the extensive school is close to the freehand brushwork. The former mostly uses sfumato and stain, and the latter mostly uses halftones and emphasizes the use of bone method and wielding the pen.

The traditional "mogu method" mainly includes the common methods such as sfumato, halftones, stain, color matching, contrast color, contrast powder, contrast water, color deposition, and ink deposition.

(1) sfumato -- -- that is, dyeing must be natural and even.

(2) Halftones -- -- spot, that is the small water droplet. It means that the color needed is spotted by calligraphy and the dense, the light, the dry and the wet must be spotted with one brushstroke.

(3) Ink matching, color matching-- -- also known as dye matching, that is, when dying separately, the two colors are connected to each other, resulting in uniform moisture and natural softness.

(4) Contrast color -- -- Contrast color is the color joint by two or more colors. When the color and the color collide, there is more moisture content, and the water is flushed to contrast color. Generally, it will write at one stretch, which can have a lively effect.

Although the mogu painting is simpler than delineation and coloring method, it is not easy to make it. Due to the short creative time, the author is more required to familiarize himself with the objects he is painting. He must have a deeper understanding, feel deeper, and have strong expressive force to make the picture vivid. Therefore, the painter must concentrate and have a well-thought-out plan when painting. Therefore, the mogu method is not easier than delineation and coloring method, and the latter can be completed in months and months, while the mogu painting is finished instantly.

\section{Contemporary Mogu Flower-bird Painting}

The contemporary mogu flower-bird painting mainly has two forms: one is the creation of a pure 
mogu flower-bird painting, and the works are mainly done using mogu techniques; the other is in the form of fine brushwork, but a large number of mogu methods are used in the painting to assist creation. Many contemporary painters have integrated the western painting theories on the basis of inheriting the traditional mogu methods and absorb other painting techniques to create new art forms. These creations are different from traditional mogu flower-bird paintings whether in materials, painting materials, or formal languages, which not only has enriched the performance techniques of flowers and birds painting, but also formed a new visual effect over the years for western modern style.

Compared with the traditional mogu flower-bird painting, the theme of the contemporary mogu flower-bird painting is more extensive and closer to life. In addition to the well-known plum, orchid, bamboo and chrysanthemum, landscapes and objects in everyday life can be painted, which can present the beauty of life in detail. For example, some painters pay more attention to the theme exploration and painting from life. They have wide painting materials and can break through the traditional theme of the performance of flower and bird painting to use a simple perspective to observe life and express the work with a simple technique. All kinds of fruits and vegetables, rocks and flowers, chickens and beasts can enter the painting. And they are very good at expressing the arrangement and texture of the image, and achieve the effect that traditional mogu flower-bird painting can't express.

Contemporary mogu flower-bird painting also emphasizes the modern composition. In the composition of the screen and the formal language organization, the contemporary painter incorporates the knowledge of modern composition on the basis of inheriting the layout rules of Chinese painting, integrates different art forms and languages of the east and the west, presents his own works with the picture that is more suitable for contemporary public aesthetics and expresses the inner spirit of the Chinese nation under the collision of multicultural art. For example, the composition and expression language in western painting is used and the western modern color expression theory and painting materials are introduced at the same time to make new explorations in the form and language to create a unique style and mood of the contemporary flowers and birds painting.

The combination of ever-changing ideas and aesthetic tastes will also make the contemporary mogu flower-bird painting show a strong sense of the times. For example, the impressionism's color principles are used to make the picture lively and vivid, or the nourishment is drawn from exotic patterns, and the modern aesthetics are contrasted boldly. These concepts are all very good examples for the formation of different styles of contemporary mogu flower-bird painting.

The introduction of new materials and new techniques. With the development of science and technology, new pigments have been continuously researched and developed. The materials that Chinese paintings can use increase gradually, which is no longer confined to the traditional water color pigment and the stone color pigments, but also includes modern rock color, watercolor, gold-silver foil and other water-soluble pigments. These pigments have a variety of materials and they are different in hue, color difference, and grain thickness. In the course of use, many different textures and effects are produced, which enriches the expressiveness of flower and bird paintings. In addition, materials such as coarse silk, water paper, and sketch paper and other materials are richer than traditional rice paper and silk paper. The difference in their texture and water absorption and color attachment can produce many unexpected special textures. And unlike traditional mogu flower-bird painting, the contemporary works are often large-scale works. On the basis of inheriting traditional mogu techniques, we constantly carry out the experiment and apply different materials to our own creation, which not only reflects the characteristics of various materials, but also enables various effects to achieve a unified and harmonious effect without losing the vividness.

From the above, we can see that contemporary mogu flower-bird painting draws the ideas of the color, composition, and layout of western modern paintings on the basis of traditional techniques, and combines with the painter's subjective creative thinking and aesthetic preferences to form a unique artistic style. This not only makes the expressive forms and technical innovations of the mogu flower-bird painting more and more exciting, but also adds fresh blood to the painting context 
of Chinese flower and bird painting to made new developments in Chinese flower and bird painting. In today's diversified and multi-context society, the aesthetic trends of artistic creation are becoming more and more diverse. Painters innovate and develop the mogu flower-bird painting from different angles, which is more conducive to satisfying the public aesthetics. The fast-changing progress and prosperity of the mogu flower-bird painting also reflects the full vitality and artistic spirit of this ancient painting category.

\section{References}

[1] Li Kuizheng, Li Kuizheng's Selected Works of Chinese Painting Creation and Teaching. Central University for Nationalities Press, 2009.

[2] Yun Shouping, Chinese Ancient Calligraphy and Painting Theory Series: Nan Tian's painting and calligraphy [M]. Hangzhou: Xiling Seal Society Press, 2008.

[3] Su Baiyi, Su Baiyi: Creation. Hebei Education Press, 2007.

[4] Jia Guangjian. Jia Guangjian's works and techniques - famous contemporary Chinese painting masters. Tianjin People's Fine Arts Press, 2013. 